\title{
Energy Management to Optimize the Lifetime of Wireless Sensor Network
}

\author{
Avhad Kalyani B. \\ Dept. of Computer Engineering \\ MIT College of Engineering \\ Pune, India
}

\author{
V. S. Deshpande \\ Dept. of Information Technology, \\ MIT College of Engineering, \\ Pune, India.
}

\begin{abstract}
The sensors in the wireless sensor network are resource constraint devices. The sensors are powered by batteries which is the most critical resource with the sensors. The limited battery limits the lifetime of the entire network. Due to more workload, some nodes die quickly which can affect the connectivity and coverage of the network. So the residual energy with remaining nodes gets wasted. Reduction in unnecessary energy consumption, clustering, mobility, use of proper transmission policy and proper transmission distance helps to reduce the wastage of energy. This paper studies such strategies that reduce the power consumption and ultimately improve the network lifetime and in next section we propose an energy aware protocol for wireless sensor network.
\end{abstract}

\section{Keywords}

wireless sensor network, energy, network lifetime, mobile node.

\section{INTRODUCTION}

The wireless sensor network is the collection of sensors which communicates each other by sending data. These sensors are very small devices distributed in a large geographical area called sensing field. It has sensing ability to sense the environmental condition; processing ability and also has some memory to store the data. It can send and receive the data within some particular communication range. The job of the sensors is to sense the environmental condition, process the data and send that information to base station. There are different types of sensors such as seismic, thermal, visual and infrared sensors. These sensors monitor variety of conditions such as temperature, humidity, characteristic of object and their motion. These different types of sensors are used in number of application like fire or flood detection, smoke detectors, measuring temperature, humidity in the air, vehicular movement, pressure, soil makeup, noise levels or mechanical stress levels. It can also be used in military, health, chemical processing and disaster, relief scenarios. Many of the home appliances like refrigerators or vacuum cleaner also includes sensors.

There are different issues related to the sensor network. For example- orderly transmission, flow and congestion control, loss recovery, quality of service, fairness, reliability, energy efficiency. Congestion is a major issue on which the rate of energy consumption depends. If congestion in the network increases, buffer of the node get full. This results in packet drop. So sender retransmits those dropped packets. This means all of the nodes which are in path from source to destination consume some energy in the retransmission of those packets. The energy requirement is more in such applications where reliability requirement is more [3].
Different protocols are available to address these different issues. Much more work has been done till date. Handling all these issue is important. But there is no single technique or protocol to address all these issues. So it is totally depend on the application which protocol to use.

The sensors are powered by batteries. Small sensors have small battery which is limited. The sensors use the energy in sensing the environment, processing that data and transmitting it to the sink node as well as forwarding the packets which are received from other nodes. Actually, the energy requirement and consumption of different nodes in network is different. The near sink node forwards more traffic towards base station so depletes energy earlier than others. Whereas the nodes far away from sink have to forward only its own data, so require less energy. Sensors having the highest rate of energy usages per data cycle are denoted as critical nodes [2] and these critical nodes limit the overall network lifetime. Lifetime of the network depends on the sensor lifetime which can be defined in different ways for example the time when first sensor runs out of energy, when first region get disconnected, when certain percentage of sensors dies or as the time when all the sensors runs out of energy [1]. The limited battery limits the lifetime of the entire network. If any sensor lost its energy, other nodes nearby that node starts depleting energy. This can cause in loss of network connectivity, coverage and reliability of the network, availability of data. The energy of the nodes get consumed more by different reasons for example retransmission due to any failure like link failure, congestion or buffer full as well as improper transmission policies, less or no cooperation between the nodes and improper node deployment strategies. If the nodes are far away from each other, then also it requires more energy in data transmission. This improper energy utilization and imbalanced energy consumption can directly affect the network lifetime. It also causes the wastage of residual energy. Many times the amount of residual energy is much more. In [8], the authors studied the non-uniform property of each node's energy consumption and residual energy consumption of the sensor networks. So to improve the sensor network lifetime, the precious resources should be saved and consumed properly.

Sometimes, the sensors can have rechargeable batteries which can be charged through the natural aspect like solar energy or it can be replaced. This battery recharging technique is called energy scavenging. It is a good way to overcome the problem of limited battery. But many times these sensors are used in such applications where it is difficult to change or recharge the battery. And that's why the sensor network lifetime is still a critical issue. Much more work is available in the literature related to reducing energy consumption and increasing the lifetime of the network. Different techniques are available to reduce the energy consumption of the nodes. This paper 
studies such strategies that reduce the power consumption and ultimately improve the network lifetime. There are some easy methods to decrease the energy used for communication such as reducing the amount of data that is transmitted, reducing the number of reporting sensors and shortening the communication range [4]. As the energy requirement of nodes is different, if we assign the energy according to requirement, then it is possible to avoid the wastage of residual energy. The non-uniform energy assignment achieves both energy efficiency and energy balance simultaneously [1]. But it is very difficult to calculate energy requirement of nodes and assign accordingly.

\section{RELATED WORK}

The sensors are cooperative in nature. This nature can be made advantageous in sensor network. In [6], authors proposed a data transmission policy namely Energy-Efficient Cooperative Communication (EECC). This policy takes advantage of cooperative nature of sensors for energy efficient transmission.

Topology: The topology of a network depends on different factors like nodes mobility, failure and duty cycle, directional antennas, and transmission power [12]. There are different topologies and different routing strategies available that can achieve energy efficiency and balanced energy consumption. In [12], authors compared balanced and progressive topology for sensor network. Both of the balanced and progressive topology provides energy efficiency but which to use depends upon network size.

Routing: The topology of network affects routing. In literature, many protocols are proposed on energy efficient routing. In [13], energy balanced routing policy is proposed. Sometimes due to uneven deployment, network may have holes. Authors, in [14] proposed a protocol that provides energy efficient routing for sensor network with holes.

Mobility: By keeping some nodes mobile, the amount of energy that is consumed in data transmission can be reduced. When the network is static i.e. all the nodes are placed on fixed position, all of the data is forwarded to sink node by the same path. So only those nodes which are on the route consume more energy than other nodes. Also the sensor network is having convergent nature. All nodes forward traffic towards the sink node. So nodes which are near to the sink have more energy consumption than others. If the nodes are mobile, the route for data will change. If the sink node is mobile, it can move and collect the data from sensors itself. This reduces the number of hops for data toward sink and balance the energy consumption of all of the nodes. In [5], authors proposed a communication strategy which can reduce this delay. Instead of mobile sink or mobile nodes, authors used a collector node which is mobile. This node moves and collects data from sensors. When this mobile data collector comes within the range of sink node, it forwards collected data to sink.

Duty cycling: If the sensor nodes have periodic job i.e. not continuous in nature then there is no use of putting the node on. To off the node when it is of no use is also an option to reduce wastage of energy. The node should be on only when there is data to be sent or receive. When the job is over, put the node in low power mode or off. This technique of energy saving is called duty cycling. But in this technique, coordination in sensors sleep and wakeup times is necessary.

The authors Hesham Abusaimeh and Shuang-Hua Yang proposed a scheme to balance the energy consumption of the network in [7]. Herein when node has energy consumption speed more than threshold value is put off. Sensor which has data to send, first check the energy consumption speed of next hop node. If it is more than the threshold value, the next hop node is put off and looks for new route for data transfer. By putting the node off for certain period of time, this scheme achieves balanced energy consumption.

Adaptive sampling: As we know, the sensors consume energy in sensing and transmission of data; so more data to send, consume more energy. Sensors sense and transmit the sensed data periodically. The network lifetime can be increased by reducing the traffic generation rate. Many times, the data to be sent is same in periodic transmission. Here is the wastage of energy which it consumed in transmission of repeated packets. To avoid this, sampling can be done. An efficient adaptive sensing and adaptive sampling strategy reduces the number of samples which in turn reduces the amount of data to be processed and transmitted [9].

Transmission policy: Like the amount of data to be transmitted, the transmission range also affects the energy consumption of node. More energy is consumed in transmitting data in long distance. So energy consumption is reduced by reducing transmission range. Varying transmission range over time attains more uniform traffic and energy usage distribution among sensors [2]. There are different transmission policies like single hop, multi hop, hybrid transmission policy. In [2], authors A. M. K. Azad and Joaeder Kamruzzaman proposed 3 different transmission policies namely Fixed hop size (FHS), Synchronous variable hop size (SVHS), Asynchronous variable hop size (AVHS). It can achieve uniform energy distribution in network.

Deployment strategy: One factor affecting the energy is deployment strategy i.e. how the nodes are deployed in network. The deployment strategy changes according to some parameters like number of sink nodes in the network, node mobility, power assignment of the sensor, placement or location of nodes, mobility options i.e. whether nodes and/or sink is static or mobile, traffic pattern. By varying these parameters the strategy can be change. Authors Zhao Cheng et al. proposed and evaluated the performance of different deployment strategies in [1].Each of the strategy has its own advantages and disadvantages but which strategy to use is totally depending on application.

Clustering: The sensors are grouped into clusters. Each cluster has assigned a node called cluster head. Each individual node within cluster transmits to cluster head instead of transmitting to sink node directly. This cluster head aggregates the data, reduce redundancy in data and then transfer data to base station. The clustering has several benefits like reduced redundancy, reduce data to transfer. This saves battery of cluster head. Data is transmitted to cluster head so the transmission range of sensors is reduced reducing energy of sensor. Generally, node that is rich in resources is selected as cluster head. The role of cluster head can be rotated over all nodes in network. This can help to achieve balanced energy consumption. In [11], authors proposed energy efficient fair clustering scheme. Here in the cluster head node is at center of cluster. In [10], authors proposed an energy efficient clustering scheme. In which, for each data collection round, probability scale i.e. the ratio of initial energy level to the average initial energy of the network is calculated. With these values, the cluster head candidates are selected. For each data collection round, node with higher resources is chosen for data transmission. 
Multiple base stations: By increasing number of sink nodes in network will help in data transmission. This can reduce transmission distance of sensors i.e. number of hops, reduce burden other nodes up to some extent. Also the chances of retransmission can be reduced.

\section{PROPOSED WORK}

Here we propose an energy efficient protocol for wireless sensor network with mobile nodes. The sensor network has convergent nature. Many nodes sense and forward data to a node which forwards towards base station. So this node i.e. forwarder node consume more energy and depletes earlier. Many times, the sensor network topology changes time to time. So it difficult to predict which node will consume more energy and which will consume less. So the idea of our proposed work is to use a mobile node to alleviate the load of the node whose energy consumption speed is more.

For example, consider the network scenario as shown in figure 1. In figure, all sensor nodes are transmitting data to sink node. Here node $n$ is having energy consumption speed higher than other nodes due to more work load. This increases imbalance energy consumption of the network. The node having more energy consumption speed i.e. $n$ will die earlier than others.

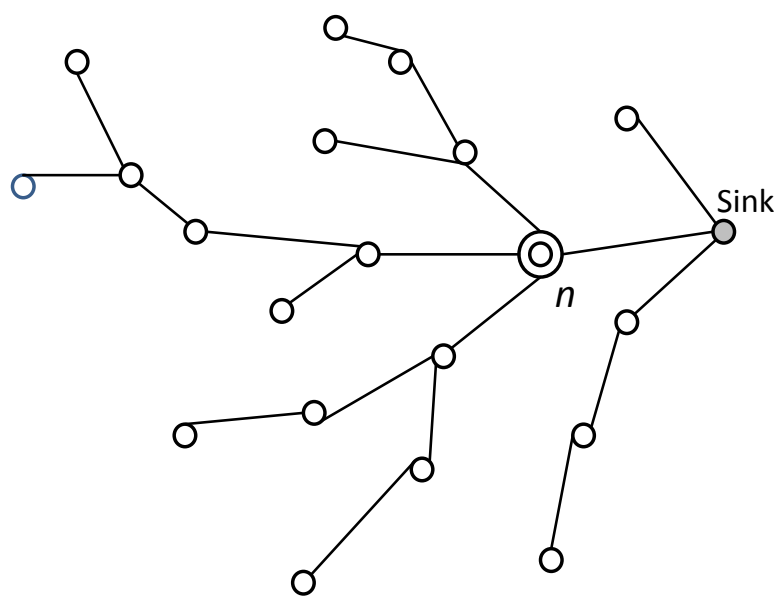

Fig. 1 Sensor network model

So to balance the energy consumption of all nodes, the consumption speed of node $n$ should be reduced. We propose idea to use mobile node nearby node $n$. This mobile node transfer data and reduce the burden of node $n$. figure 2 shows the pseudo code for our work.

\section{Algorithm}

1. To check the status of all nodes. Initially we have to check status of nodes means energy consumption speed of nodes.

2. Check the threshold value for energy. According to the energy consumption status of nodes, we will set the threshold value for the energy.

3. Controlling the cooperative node movement.

Then the coordinator node should be moved to the area of the node whose energy consumption speed is more than others. That means find location for the cooperative node.

4. Check the performance parameters again.
Initially, the sender of data checks the energy consumption speed of next hop node. If this speed exceeds certain threshold, then cooperative node is called within that region. Sender transmits the traffic through this cooperative node. If the energy consumption speed do not exceed, the sender of data transmit to the next hop node. Figure 3 shows the flowchart for the proposed scheme. We are going to evaluate the performance of the proposed scheme. In our future work, we will implement and find out the results of above mentioned protocol.

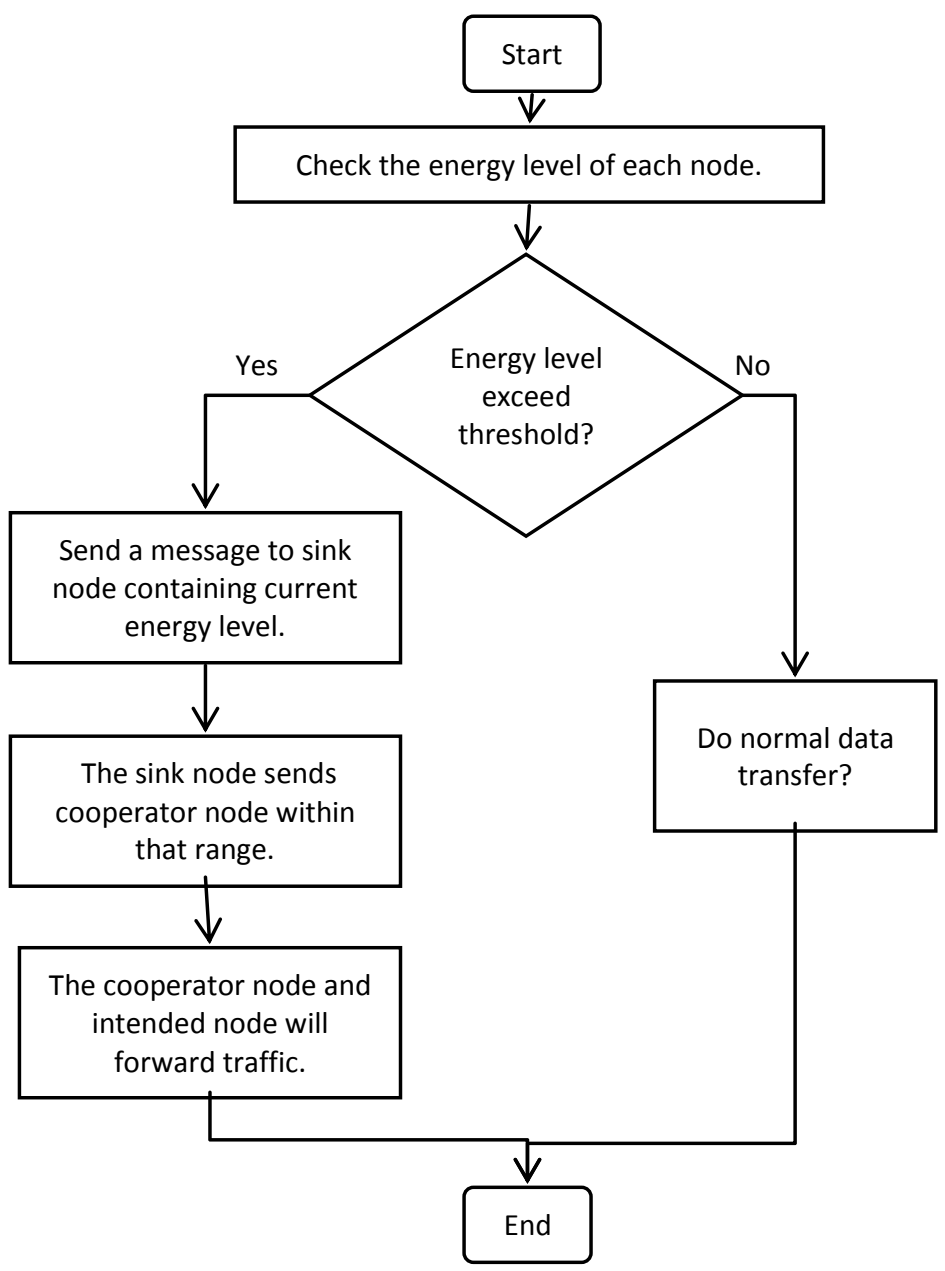

Fig. 3 Flowchart for proposed protocol

\section{CONCLUSION}

The energy of sensor nodes in the sensor network is very precious resource. It is difficult to recharge the battery of the sensors. More energy consumption speed of a single node can imbalance the energy consumption. This can directly affect the entire lifetime of the entire network. So the energy should be consumed in proper manner. The balanced energy consumption of the network definitely increases the network lifetime. The congestion in the network causes the packet loss and retransmission which results in wastage of the energy of the sensors. The use of mobile nodes that can be sink node or simple forwarder node in the network also helps to data transfer. This alleviates the lode of the sensors and lessens the chance of congestion. So in this way, use of mobile nodes, cooperative nodes, proper deployment strategies and the scheme to balanced power consumption speed, transmission policies and also clustering policies can reduce the energy consumption and increase the network lifetime.

Fig. 2 Algorithm for proposed technique 
The future work is to implement the above mentioned protocol i.e. using the mobile node and evaluate the results of it.

\section{REFERENCES}

[1] Zhao Cheng, Mark Perillo, and Wendi B. Heinzelman, "General Network Lifetime and Cost Models for Evaluating Sensor Network Deployment Strategies", IEEE Transactions On Mobile Computing, Vol. 7, No. 4, April 2008.

[2] A.K.M. Azad and JoarderKamruzzaman, "EnergyBalanced Transmission Policies for Wireless Sensor Networks", IEEE Transactions On Mobile Computing, Vol. 10, No. 7, July 2011.

[3] D. Q. Bai, In-Yeup Kong and Won-Loo Hwang, "Tradeoff between Reliability and Energy-efficiency in Transport Protocol for Wireless Sensor Networks", SICE-ICASE International Joint Conference, pp. 429 434, Oct. 18-21, 2006

[4] Zhi Tan, Yun Liu, Zhenjiang Zhang, "Performance Requirement on Energy Efficiency in WSNs",3rd International IEEE Conference on Computer Research and Development (ICCRD), Vol. 3, pp. 159-162, March 2011.

[5] Arun K. Kumar and Krishna M. Sivalingam, "EnergyEfficient Mobile Data Collection in Wireless Sensor Networks with Delay Reduction using Wireless Communication",Second International IEEE Conference on Communication Systems and Networks, pp. 1-10, January 2010.

[6] Weiwei Fang, Feng Liu, Fangnan Yang, Lei Shu, and ShojiroNishio, "Energy-Efficient Cooperative Communication for Data Transmission in Wireless Sensor Networks", IEEE Transactions on Consumer Electronics, Vol. 56, No. 4, pp. 2185-2192, November 2010.

[7] HeshamAbusaimeh and Shuang-Hua Yang, "Balancing the Power Consumption Speed in Flat and Hierarchical WSN", International Journal of Automation and Computing, Vol. 05, No. 4, pp. 366-375, October 2008.
[8] Yang wenguo and GuoTiande, "The Non-uniform Property of Energy Consumption and its Solution to the Wireless Sensor Network", Second International IEEE Workshop on Education Technology and Computer Science, Vol. 2, pp. 186-192, 2010.

[9] CesareAlippi, GluseppeAnastasi, Mario Di Francesco and Manuel Roveri, "Energy Management in Wireless Sensor Networks with Energy-Hungry Sensors", IEEE Instrumentation and Measurement Magazine, Vol. 2, issue. 2, pp. 16-23, April 2009.

[10] Dali Wei, Yichao Jin, SerdarVural, Klaus Moessner, and Rahim Tafazolli, "An Energy-Efficient Clustering Solution for Wireless Sensor Networks", IEEE Transactions On Wireless Communications, Vol. 10, No. 11, November 2011

[11] Sungjin Park, Woongsup Lee, and Dong-ho Cho, "Fair Clustering for Energy Efficiency in a Cooperative Wireless Sensor Network", $75^{\text {th }}$ IEEE Conference on Vehicular Technology, pp. 1-5, 2012.

[12] Maneesha V Ramesh1, Sreedevi A. G2, KamalanathanK3, P VenkatRangan, "Energy Comparison of Balanced and Progressive Sensor Networks", Annual IEEEConference on Wireless and Optical Communications, pp. 93-98, April 19-21, 2012.

[13] FengyuanRen, Jiao Zhang, Tao He and Sajal K. Das, "EBRP: Energy-Balanced Routing Protocol for DaaGatherig in Wireless Sensor Network", IEEE Transactions on Parallel and Distributed Systems, Vol. 22, No. 12, December 2011

[14] Shiow-Fen Hwang, Hsin-Hui Lin, Chyi-Ren Dow, “An Energy-Efficient Routing Protocol in Wireless Sensor Networks with Holes", Fourth International Conference on Ubiquitous and Future Networks, pp. 17-22, 2012.

[15] I.F. Akyildiz, W. Su, Y. Sankarasubramaniam, E. Cayirci, "Wireless sensor networks: a survey", Computer Networks, pp. 393-422, 2002. 